\title{
Fisiologia da Circulação Fetal e Diagnóstico das Alterações Funcionais do Coração do Feto
}

\author{
Sandra S. Mattos \\ Recife, PE
}

$\mathrm{O}$ feto cardiopata necessita de cuidados especiais. O útero materno, na maioria das vezes, é a sua melhor UTI. Neste, a temperatura ambiente é constante, nutrição parenteral é administrada com mínimos riscos, não há manuseio reduzindo a incidência de trauma e infecção, e, principalmente, no tocante ao aparelho cardiovascular, ele é mantido em circulação extracorpórea, utilizando o mais eficiente e seguro oxigenador de membranas: a placenta. A compreensão da fisiologia da circulação feto-materno-placentária é de fundamental importância na avaliação do comportamento das diversas alterações do sistema cardiovascular na vida intra-uterina.

\section{Fisiologia da circulação fetal}

A circulação fetal difere da extra-uterina anatômica e funcionalmente. Ela é estruturada para suprir as necessidades de um organismo em crescimento rápido num ambiente de hipóxia relativa. A única conexão entre o feto e o meio externo é a placenta, que o serve nas funções de "intestinos" (suprimento de nutrientes), "rins" (retirada dos produtos de degradação) e "pulmões" (trocas gasosas).

Os pulmões fetais estão cheios de líquido, oferecendo alta resistência ao fluxo sangüíneo. A placenta contém grandes seios venosos, funcionando como uma fístula arteriovenosa com baixa resistência ao fluxo sangüíneo sistêmico. Enquanto que na vida extra-uterina os ventrículos trabalham em série, com o débito cardíaco do ventrículo direito (VD) igualando aquele do esquerdo, no feto, através de quatro bypasses principais - o foramen oval, o canal arterial, a placenta e o ducto venoso, os ventrículos trabalham em paralelo. O sangue oxigenado proveniente da placenta chega ao feto através da veia umbilical. Esse sangue passa principalmente (45\%) através do ducto venoso "bypassando" o fígado fetal ${ }^{1}$. O sangue venoso portal se mistura com este e, conseqüentemente, o sangue da veia cava inferior é menos saturado do que o sangue da veia umbilical. Ainda assim, com aproximadamente $70 \%$ de saturação de $\mathrm{O}_{2}$, esse sangue é o mais oxigenado de todo o retor-

Unidade de Cardiologia e Medicina Fetal do Hospital Português - Recife Correspondência: Sandra S. Mattos - Av. Agamenon Magalhães S/N - 50090-900 - Recife, PE no venoso, tendo a veia cava superior uma saturação de aproximadamente $40 \%{ }^{2}$. O sangue da cava inferior representa aproximadamente $70 \%$ do volume total do retorno venoso. Este chega ao átrio direito $(\mathrm{AD})$ e é parcialmente (33\%) dirigido para o átrio esquerdo (AE) através do foramen oval. A energia cinética do fluxo sangüíneo da veia cava inferior é a principal responsável pela manutenção da perviabilidade do foramen oval no feto, já que as diferenças nas pressões médias da veia cava, $\mathrm{AD}$ e $\mathrm{AE}$ são mínimas ${ }^{3}$. O restante do fluxo de retorno da cava inferior mistura-se ao retorno da veia cava superior e seio coronário e passa para o VD. O sangue que chega ao AE e daí ao ventrículo esquerdo e a aorta ascendente, artérias coronárias e cérebro é, conseqüentemente, o mais saturado com aproximadamente $65 \%$ em relação a uma saturação de $55 \%$ no VD, que será dirigido através do canal arterial para a parte inferior do corpo do feto ${ }^{4}$. O istmo da aorta recebe apenas $10 \%$ do débito cardíaco total e, pelo seu estreitamento fisiológico, "separa" o fluxo entre a aorta ascendente e a descendente ${ }^{2}$. O baixo fluxo pulmonar fetal é mantido às custas da elevada resistência vascular pulmonar. Vários fatores estimulam esta vasoconstricção como acidose, catecolaminas alfa-adrenérgicas e estimulação nervosa simpática, porém não há dúvidas que a

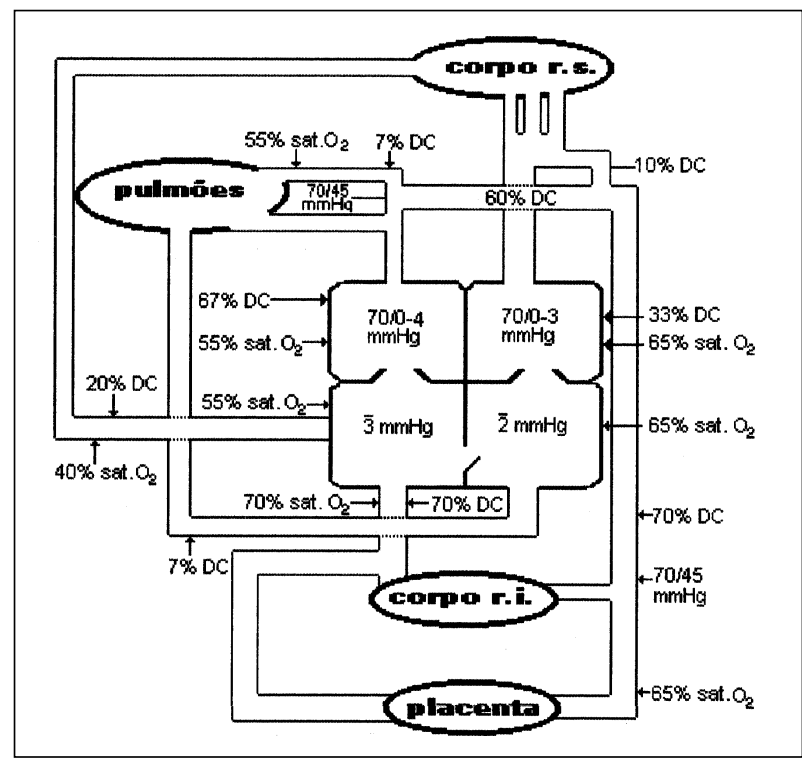

Fig. 1 - Diagrama da distribuição do volume sangüíneo, níveis pressóricos e de saturação de oxigênio fetais. Adaptado ${ }^{6,7}$. 


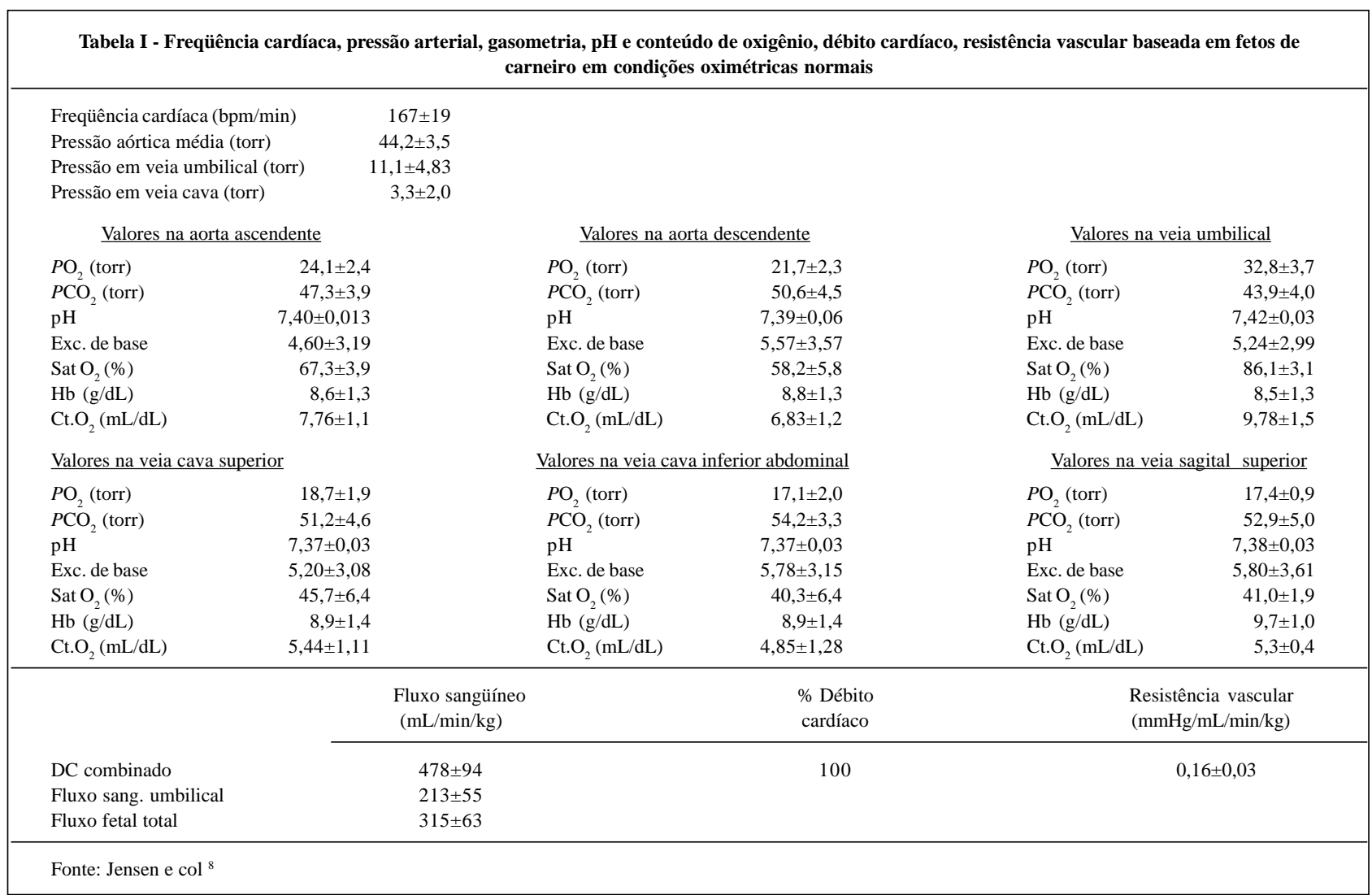

hipóxia é o principal fator determinante da vasoconstricção pulmonar fetal. Devido à alta resistência ao fluxo sangüíneo pulmonar, apenas uma pequena quantia (aproximadamente $7 \%$ do débito cardíaco combinado) de sangue circula pelos pulmões, o restante é dirigido, através do canal arterial para a aorta descendente. Embora o VD (60\%) apresente um débito cardíaco superior ao do $\mathrm{VE}(40 \%)$ na vida intra-uterina, há evidências anatômicas e ecocardiográficas de que o desenvolvimento destas cavidades são semelhantes durante toda a gestação ${ }^{5}$.

A figura 1, adaptada de estudos realizados em fetos de carneiro por Rudolph e $\mathrm{col}^{6}$, e Heyman e $\mathrm{col}^{7}$, mostra esquematicamente a distribuição do volume sangüíneo, os níveis pressóricos e de saturação de oxigênio fetais. Na tabela I, adaptada também de estudos em fetos de carneiros ${ }^{8}$ estão sumarizados freqüência cardíaca, pressão arterial, gasometria, pH, conteúdo de oxigênio, e, ainda, a distribuição sangüínea para os órgãos, débito cardíaco, liberação de $\mathrm{O}_{2}$ e resistência vascular fetais.

\section{Diagnóstico das alterações funcionais do coração do feto}

Com o conhecimento da fisiologia da circulação fetal, podemos facilmente entender os diferentes comportamentos das alterações do sistema cardiovascular fetal que nos levam a subdividir as cardiopatias fetais em ativas e passivas. Incluem-se sob o grupo de cardiopatias passivas, todas as malformações do sistema cardiovascular fetal que não provocam repercussão hemodinâmica intra-útero, notadamente as cardiopatias estruturais, sem regurgitação valvar. Muitas cardiopatias graves, canal-dependentes, também se incluem neste grupo, por só manifestarem alterações clínicas no período neonatal, após o fechamento do canal arterial. O outro grupo, ao qual chamamos de cardiopatias ativas, inclui todas as alterações do sistema cardiovascular fetal que provocam repercussão hemodinâmica ainda na vida intra-uterina. Neste, incluímos, as arritmias cardíacas, as cardiopatias estruturais com regurgitação valvar importante que podem evoluir para insuficiência cardíaca fetal, miocardiopatias e miocardites, e as alterações funcionais do coração fetal como resposta a condições adversas. Neste último grupo, destacamos a hidropisia não imune, o crescimento intra-uterino retardado, o diabetes mellitus e a hipertensão arterial materna, a anemia fetal e o uso de drogas.

O principal fator de descompensação cardiovascular fetal, comum a todas as situações acima é a hipóxia. Nesta situação, independente de seu mecanismo etiológico há uma redistribuição do fluxo sangüíneo fetal, que pode ser resumida como: 1) um aumento do fluxo cerebral e adrenal;2) uma diminuição do fluxo sistêmico conseqüente a aumento da resistência vascular sistêmica (pós-carga); e 3) um aumento na proporção do sangue venoso umbilical que entra 
o ducto venoso, com aumento do fluxo para o lado esquerdo do coração.

Sob o ponto de vista funcional, as principais respostas do sistema cardiovascular fetal a situações adversas são a dilatação, o aparecimento de efusão pericárdica ${ }^{9} \mathrm{e}$ a perda da função contrátil. Cardiomegalia com ou sem derrame pericárdico é um importante sinal de disfunção cardíaca fetal ${ }^{10}$. A presença e severidade de efusão pericárdica é um dado importante de disfunção cardíaca fetal que também pode ser avaliado com a ecocardiografia bidimensional.

Do ponto de vista hemodinâmico, alterações da précarga ou intrínsecas do miocárdio ventricular como àquelas observadas em fetos de mães diabéticas podem alterar a evolução normal do enchimento ventricular, prolongando o período de dominância da contração atrial até após o nascimento ${ }^{11}$. Estes achados podem ser mais evidentes no lado direito do coração por ser o volume de fluxo transvalvar tricuspídeo maior que o mitral durante toda a gestação ${ }^{12}$.

A utilização criteriosa da ecocardiografia na avaliação do fluxo intra e extracardíaco fetal pode detectar precocemente estas alterações. Esta abordagem precoce vem assumindo um importante papel no manuseio de gestações patológicas como as acima citadas e reitera a importância do trabalho multidisciplinar entre obstetras, fetologistas e cardiologistas fetais no rastreamento e manuseio das alterações do sistema cardiovascular fetal.

\section{Referências}

1. Elderstone DI, Rudolph AM,Heyman MA-Effects of hypoxemia and decreasing umbilical flow on liver and ductus venosus blood flow in fetal lambs. Am J Physiology 1980; 238: H656.

2. Rudolph AM, Heymann MA - The circulation of the fetus in utero. Methods for studying blood flow, cardiac output and organ blood flow. Circ Res 1967; 26: 289.

3. Anderson D, Faber J, Morton M, Parks C, Pinson C, Thornburg K - Flow through the foramen ovale in the fetal newborn lamb. J Physiology 1985; 365:19.

4. Elderstone DI, Rudolph AM. Preferential streaming of the ductus venosus blood to the brain and heart in fetal lambs. Am J Obstet Gynecol 1979; 237: H724

5. St John Sutton MG, Raichlen JS, Reichek N, Huff DS - Quantitative assessment of right and left ventricular growth in the human fetal heart: a pathoanatomic study. Circulation 1984; 70: 935.

6. Rudolph AM, Heymann MA - Control of Fetal Circulation. In: Comline RS, Dawes GS, Nathaniels PW, eds - Fetal and Neonatal Physiology. London: Cambridge University Press, 1983.

7. Heyman MA - Management of the newborn circulation. In: Jones CT, Nathanielsz
PW - The Physiological Development of the Fetus and Newborn, part 7. ed London: Academic Press Inc, 1985; pp 721-731.

8. Jensen A, Roman C, Rudolph AM - Effects of reducing uterine blood flow distribution and fetal blood oxygen delivery. J Development Physiology 1991; 15 : 309.

9. DeVore GR, Donnerstein RL, Kleiman CS, Platt LD, Hobbins JC. Fetal Echocardiography. II - Diagnosis and significance of a pericardial effusion in the fetus using real time directed M mode ultrasound. Am J Obstet Gynecol 1982; 144: 693 .

10. Johnson P, Sharland G, Allan LD, Tynan MJ, Maxwell DJ - Umbilical venous pressure in nonimmune hydrops fetalis: correlation with cardiac size. Am J Obstet Gynecol 1992; 167: 1309.

11. Rizzo G, Arduini D, Romanini C - Cardiac function in fetus of type I diabetic mothers. J Pediatr 1991; 118:103.

12. Reed KL, Sahn DJ, Scagnelli S, Anderson CF, Shenker L - Doppler echocardiographic studies of diastolic function in the human fetal heart: changes during gestation. J Am Coll Cardiol 1986; 8: 381 